\title{
Integrated analyses of DNA methylation and hydroxymethylation reveal tumor suppressive roles of ECM1, ATF5, and EOMES in human hepatocellular carcinoma
}

\author{
Fei Gao ${ }^{1^{* \dagger}}$, Yudong Xia ${ }^{1 \dagger}$, Junwen Wang ${ }^{1}$, Zhilong Lin ${ }^{1}$, Ying Ou ${ }^{2,3}$, Xing Liü ${ }^{2,3}$, Weilong Liu, ${ }^{4,5}$, Boping Zhou, ${ }^{4,5}$ \\ Huijuan Luo', Baojin Zhou' ${ }^{1}$, Bo Wen ${ }^{1}$, Xiuqing Zhang ${ }^{1}$ and Jian Huang ${ }^{2,3,5^{*}}$
}

\begin{abstract}
Background: Differences in 5-hydroxymethylcytosine, 5hmC, distributions may complicate previous observations of abnormal cytosine methylation statuses that are used for the identification of new tumor suppressor gene candidates that are relevant to human hepatocarcinogenesis. The simultaneous detection of 5-methylcytosine and 5-hydroxymethylcytosine is likely to stimulate the discovery of aberrantly methylated genes with increased accuracy in human hepatocellular carcinoma.

Results: Here, we performed ultra-performance liquid chromatography/tandem mass spectrometry and single-base high-throughput sequencing, Hydroxymethylation and Methylation Sensitive Tag sequencing, HMST-seq, to synchronously measure these two modifications in human hepatocellular carcinoma samples. After identification of differentially methylated and hydroxymethylated genes in human hepatocellular carcinoma, we integrate DNA copy-number alterations, as determined using array-based comparative genomic hybridization data, with gene expression to identify genes that are potentially silenced by promoter hypermethylation.

Conclusions: We report a high enrichment of genes with epigenetic aberrations in cancer signaling pathways. Six genes were selected as tumor suppressor gene candidates, among which, ECM1, ATF5 and EOMES are confirmed via siRNA experiments to have potential anti-cancer functions.
\end{abstract}

\section{Background}

Hepatocellular carcinoma (HCC), which is frequently caused by hepatitis virus (B and C) infection and alcohol abuse, is the most common type of primary liver cancer and third leading cause of cancer death worldwide $[1,2]$. Although surgical and chemotherapeutic treatment of $\mathrm{HCC}$ is evolving, surgical resection remains the treatment of choice for many patients. Surgical resection for HCC patients is associated with a 5-year survival rate of 50\%; however, there is a $70 \%$ recurrence rate [3].

\footnotetext{
*Correspondence: flys828@gmail.com; huangjchgc@hotmail.com ${ }^{\dagger}$ Equal contributors

'BGI-Shenzhen, Shenzhen 518083, China

${ }^{2}$ National Engineering Center for Biochip at Shanghai, Shanghai 201203,

China

Full list of author information is available at the end of the article
}

The mechanism underlying HCC development remains poorly understood. It is widely accepted that accumulating genetic alterations such as chromosomal alterations, gene amplifications, and mutations are associated with HCC $[4,5]$. Furthermore, epigenetic alterations, particularly abnormal DNA methylation at the 5 position of cytosine $(5 \mathrm{mC})$, have been extensively studied [6]. DNA hypomethylation in cancer cells is thought to lead to chromosomal instability and oncogene activation [7] and has generally been regarded as a highly stable clinical marker for cancer [8]. More importantly, a number of studies have reported that the hypermethylation of tumor suppressor genes (TSGs) contributes to HCC pathogenesis [9-11]. Thus, the accurate detection of DNA methylation may provide powerful mechanistic insight into hepatocarcinogenesis and may have a potential application for the clinical diagnosis of HCC. 
However, the differences in 5-hydroxymethylcytosine $(5 \mathrm{hmC})$ distributions may complicate previous observations regarding abnormal cytosine methylation status. Previous technologies, such as bisulfite treatment and restriction enzyme-based technologies, are unable to distinguish between $5 \mathrm{mC}$ and $5 \mathrm{hmC}[12,13]$, and the existence of $5 \mathrm{hmC}$ in samples reduces the accuracy of DNA methylation detection [14]. $5 \mathrm{hmC}$ is catalyzed by teneleven translocation (TET) proteins, which first convert $5 \mathrm{mC}$ to $5 \mathrm{hmC}$, then to 5 -formylcytosine $(5 \mathrm{fC})$ and finally to 5 -carboxylcytosine $(5 \mathrm{caC})$, thereby may play a role in DNA demethylation $[15,16]$. $5 \mathrm{hmC}$ was found to be abundant in embryonic stem cells and neurons, but it is greatly reduced in tumor cells [15,17-20], including HCC cells [21].

Therefore, there is a renewed interest for the simultaneous detection of $5 \mathrm{mC}$ and $5 \mathrm{hmC}$ in the context of genomic profiling studies, which may stimulate the discovery of aberrantly methylated genes with increased accuracy in HCC cells. Till now, the number of known aberrantly promoter-methylated genes is fewer for HCC than for colon and gastric cancer $[5,6]$. Furthermore, a more comprehensive study of the $5 \mathrm{hmC}$ status in $\mathrm{HCC}$ is required to determine its role in hepatocarcinogenesis.

To fully examine $5 \mathrm{mC}$ and $5 \mathrm{hmC}$ status in $\mathrm{HCC}$, we used ultra-performance liquid chromatography/tandem mass spectrometry (UPLC-MS/MS) and a newly developed single-base high-throughput sequencing approach (hydroxymethylation and methylation sensitive tag sequencing (HMST-seq)) to synchronously measure these two modifications in HCC samples and their adjacent non-cancerous liver tissues (non-HCCs). We report a global loss of $5 \mathrm{hmC}$ and key genes containing altered methylation or hydroxymethylation that are enriched for important cancer-relevant signaling pathways. In particular, we identified three new genes (ECM1, ATF5, and $E O M E S$ ) with potential anti-cancer functions that may promote the understanding of the molecular mechanisms of HCC development and progression and potentiate the future clinical applications of $5 \mathrm{hmC}$ detection.

\section{Results}

\section{Globally increased $5 \mathrm{mC}$ but decreased $5 \mathrm{hmC}$ levels at genomic CCGG loci in HCC}

We first performed UPLC-MS/MS to investigate global $5 \mathrm{mC}$ and $5 \mathrm{hmC}$ levels in 16 pairs of HCC and non-HCC samples and two HCC cell lines (97 L and LM6 cells). We found that both $5 \mathrm{mC}$ and $5 \mathrm{hmC}$ were frequently decreased in $71 \%$ and $100 \%$ of the HCC specimens, respectively, compared with non-HCC specimens. Correspondingly, $5 \mathrm{mC}$ and $5 \mathrm{hmC}$ levels in the two $\mathrm{HCC}$ cell lines remained relatively low (Additional file 1 : Tables S2). These results are in accordance with previous reports of a global loss of $5 \mathrm{mC}$ and $5 \mathrm{hmC}$ in HCC and other cancer types $[18,20,21]$.

To further examine the distribution of $5 \mathrm{mC}$ and $5 \mathrm{hmC}$ in HCC specimens and cell lines, we applied a newly developed technology, 'HMST-seq' [22], on seven of the 16 pairs of HCC specimens and the two cell lines. HMST-seq can detect $5 \mathrm{mC}$ and $5 \mathrm{hmC}$ at 'CCGG' sites with a singlebase resolution, in which three different libraries were constructed for each sample including a library containing unmodified $\mathrm{C}, \mathrm{mC}$, and $\mathrm{hmC}$ tags through $M s p \mathrm{I}$-mediated digestion of normal genomic DNA, a library containing $\mathrm{C}$ and $\mathrm{mC}$ tags through $M s p \mathrm{I}$-mediated digestion of glucosylated genomic DNA and a library containing only $\mathrm{C}$ tags through HpaII-mediated digestion of normal genomic DNA [22]. During manual examination of the tag distribution of these three libraries, we filtered out samples with similar distributions of ' $\mathrm{C}+\mathrm{mC}$ ' tags and ' $\mathrm{C}$ ' tags, which indicate potential insufficient $\mathrm{Msp} \mathrm{I}$-mediated digestion of glucocylated genomic DNA (Additional file 2: Figure S1). As a result, three out of the seven matched pairs of HCC and non-HCC specimens passed our quality control. We then used these three matched pairs of specimens to infer methylation and hydroxymethylation status across all CCGG sites. On average, 170 million reads for each library were generated, and 147 million (86.58\%) reads were aligned to a virtual library of the human genome (see the Methods section). An average of $100 \mathrm{M}$ (58.93\%) uniquely aligned reads were then obtained for each library, resulting in a minimum of $1.3 \mathrm{M}$ informative 'CCGG' sites with greater than $50 \mathrm{X}$ sequencing depth for each sample (Additional file 1: Table S3). Data normalization for the three libraries was performed, and significantly modified sites with $5 \mathrm{mC}$ or $5 \mathrm{hmC}$ were identified with our established methods (sequencing depth $>10 \mathrm{X}, \mathrm{FDR}<0.001$ ) [22]. On average, 433,045 (31.49\%) and 72,080 (5.24\%) total examined CCGG sites were identified as significant for $5 \mathrm{mC}$ and $5 \mathrm{hmC}$, respectively, in non-HCC specimens. In addition, increased $5 \mathrm{mC}(471,270 ; 34.72 \%)$ but decreased $5 \mathrm{hmC}(66,020 ; 4.89 \%)$ was observed in the HCC specimens (Additional file 1: Table S2).

Based on these data, a broad distribution of $5 \mathrm{mC}$ and $5 \mathrm{hmC}$ was observed (Figure 1). Compared with the background distribution of all 'CCGG' sites in the genome, $5 \mathrm{mC}$ was more deficient in regulatory regions such as enhancers or regions surrounding transcriptional start sites (TSS) but relatively enriched in exons and intragenic regions. In contrast, the distribution of $5 \mathrm{hmC}$ was more evenly distributed with a background distribution across all 'CCGG' sites (Additional file 2: Figure S2). In agreement with previous reports that $\mathrm{CpG}$ islands are hypermethylated in tumors [8], globally increased $5 \mathrm{mC}$ was observed in CCGG sites across the genome of HCC compared with non-HCC samples (Figure 1A). In contrast, $5 \mathrm{hmC}$ levels of CCGG sites across the HCC genome were 

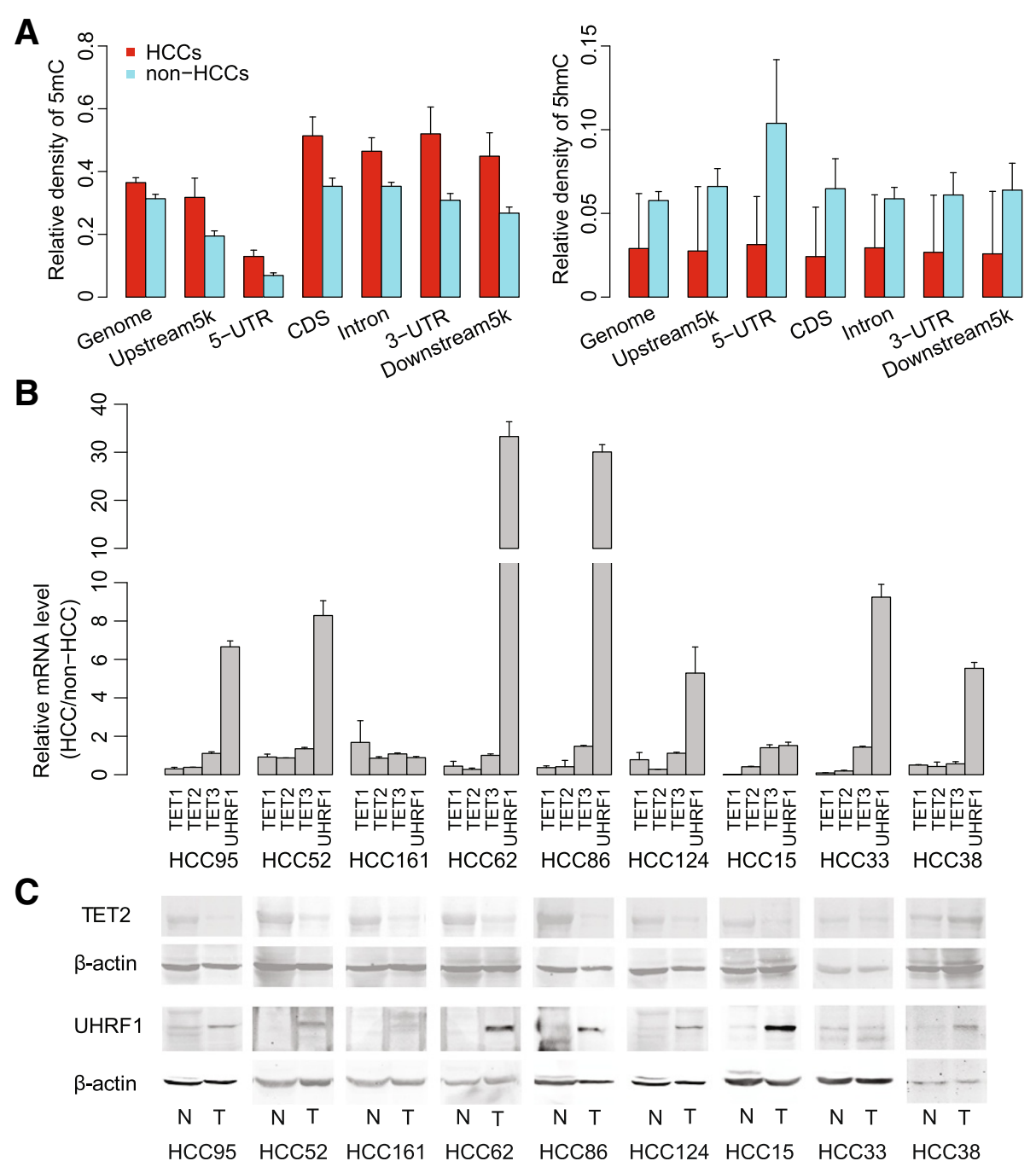

Figure 1 The relative density of $5 \mathrm{mC}$ and $5 \mathrm{hmC}$ within genomic regions. (A) The relative density indicates the ratio between $5 \mathrm{mC}$ or $5 \mathrm{hmC}$ and all inspected 'CCGG' sites within each genomic region. (B) The relative mRNA levels of UHRF1, TET1, 2, and 3 in HCCs and adjacent non-HCC liver tissues tested by RT-qPCR. (C) The expression of TET2 and UHRF1 protein in HCCs and adjacent non-HCC liver tissues using western blot. $\beta$-actin was used as loading control.

decreased, in consistence with whole-genome $5 \mathrm{hmC}$ decrease tested by UPLC-MS/MS.

As TET proteins are responsible for oxidizing $5 \mathrm{mC}$ to $5 \mathrm{hmC}[15,16]$, we tested mRNA levels of TET1, 2, and 3 in nine pairs of HCC specimens. We found downregulation of mRNA levels of TET1 and TET2, but not TET3, in HCCs in comparison with non-HCC samples (Figure 1B). Consistent with its mRNA level, TET2 protein was confirmed downregulated in HCCs as well (Figure 1C). We also tested UHRF1 as its protein helps to recruit DNMT1 to hemi-methylated DNA to facilitate faithful maintenance of DNA methylation [23]. In contrast to TET genes, both mRNA and protein expression levels of UHRF1 gene were highly upregulated in HCCs (Figure 1B and C), consistent with previous reports in various types of cancers [24]. Based on these results, expression level changes of key enzymes or cofactors that are involved in DNA methylation/hydroxymethylation regulation might be correlated with $5 \mathrm{mC}$ increase and $5 \mathrm{hmC}$ decrease in CCGG sites across the HCC genome.

\section{DMRs and DhMRs are revealed by inter-group comparisons}

Based on the observation of global methylation and hydroxymethylation alterations, we next aimed to specify differentially methylated regions (DMRs) and differentially hydroxymethylated regions (DhMRs) between HCC and non-HCC samples. A sliding window strategy was used for inter-group comparison of the HCC and non-HCC groups as previously described [22]. To identify differentially modified regions containing at least five CCGG sites (Wilcoxon rank-sum test, $P<0.05$ ), we began with the 
three matched pairs and found 1,851 DMRs and 243 DhMRs (Additional file 1: Table S4, S5). Although both modifications were widely distributed across the genome, DMRs occurred more frequently at proximal regions close to TSSs, while DhMRs were more likely to occur at distal sites upstream of TSSs (Figure 2A and B). Thus, only 10 (4.12\%) DhMRs overlapped with DMRs, suggesting that changes in these two modifications rarely co-occur.

Furthermore, we compared the $5 \mathrm{mC}$ and $5 \mathrm{hmC}$ levels of the DMRs and DhMRs, respectively, among HCC and nonHCC samples and two HCC cell lines (97 L and LM6). $97 \mathrm{~L}$ and LM6 were derived from the same HCC patient but exhibit highly different metastatic potentials [25]. Similar to the HCC samples, hypermethylation of the DMRs and hypohydroxymethylation of the DhMRs were observed in $97 \mathrm{~L}$ and LM6 compared with non-HCC cells. Notably, the LM6 cells, which have a higher metastatic potential, exhibited a slightly higher methylation level in the DMRs compared with 97 L cells (Additional file 2: Figure S3).

To replicate above results, we re-performed HMSTSeq library construction on two pairs of the samples that first failed our quality control. Similar data quality and

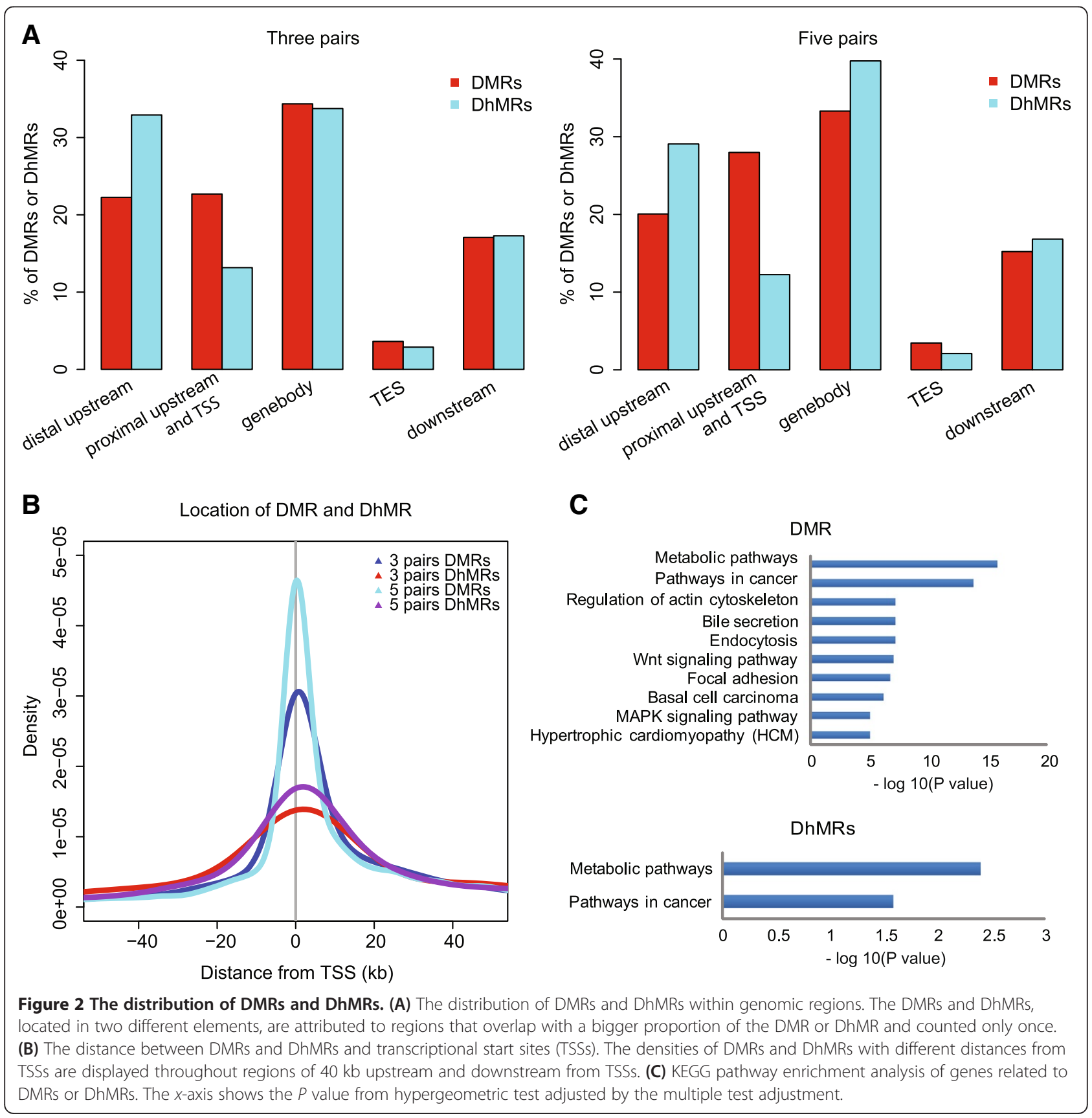


$5 \mathrm{mC}$ and $5 \mathrm{hmC}$ levels were achieved (Additional file 1 : Table S2, S3). By combining all qualified data, an intergroup comparison of five matched pairs of HCC and non-HCC samples was re-performed, resulting in a total of 5,126 DMRs and 571 DhMRs using the same window-sliding strategy (Additional file 1: Table S4, S5); similar results regarding the distribution of DMR and DhMR were observed (Figure 2A, B).

\section{Enrichment of genes containing DMRs and DhMRs in cancer-related pathways}

To further address the functional effects of DNA modification changes in HCC, we first performed KEGG pathway enrichment analysis (see the Methods section) of 1,373 DMR and 218 DhMR genes from the three matched pairs of HCC and non-HCC samples, respectively. A higher enrichment rate for DMR (16.8\%; 231 out of 1373) than DhMR genes (8.7\%, 19 out 218; FDR $<0.05$ ) was found (Additional file 1: Table S6). Although DMR and DhMR genes were enriched in KEGG cancer and metabolic pathways (Figure $2 \mathrm{C}$ ), these genes rarely overlapped (Additional file 2: Figure S4). In addition, enrichment analysis of 3,283 DMR and 651 DhMR genes from the five pairs of HCC and non-HCC samples, respectively, revealed similarly enriched pathways (data not shown). Notably, cancer-related pathways including 'Pathways in cancer', 'Focal adhesion', 'Wnt signaling pathway', and 'MAPK signaling pathway' were enriched for DMR genes.

Focal adhesion components are crucial for the cell growth, movement, differentiation, and tailoring of the extracellular microenvironment [26], and of these genes, focal-adhesion kinase $(F A K)$ is an important mediator involved in cancer formation and progression [27]. Multiple genes that encode extracellular proteins and transmit signals, including extracellular matrix (ECM), integrin, SRC, and growth factor (GF) genes, were hypermethylated in HCCs. Signaling by the Wnt family of secreted glycolipoproteins via the transcriptional coactivator $\beta$-catenin can also determine cell fate and has been implicated in cancer proliferation and survival, including HCC cells [28]. Aberrant methylation was also observed for key genes of the Wnt signaling pathway including the Wnt, FZD, and APC genes. Furthermore, multiple key genes in the MAPK signaling pathway were enriched among those containing DMRs, including TGFB2, EGFR, NFkB2, and p38 mitogen-activated protein kinase (Figure 3, Additional file 1: Table S6).

DMR genes were also enriched in metabolic pathways, which are also relevant. In particular, one gene, PKLR, was aberrantly methylated in HCC; PKLR encodes a pyruvate kinase and is involved in the glycolysis process and important for cancer metabolism and tumor growth, $[29,30]$. In summary, these results suggest that abnormal
DNA methylation and gene hydroxymethylation might be crucial participants in important cancer-related pathways in $\mathrm{HCC}$.

\section{Transcriptional changes in key genes in cancer pathways are revealed by integration of multi-omics data}

To reveal potential complications resulting from DNA modification changes on gene transcription, we further performed transcriptome profiling of the seven pairs of HCC samples and two HCC cell lines using RNA-Seq and Affymetrix microarray technologies. Based on the high-quality data, we performed pair-wise comparisons to identify differentially expressed genes (DEGs) in the seven pairs of HCC samples. Copy number variation $(\mathrm{CNV})$ analyses were then used to address transcription changes due to gene CNVs (see the Methods section). If significantly upregulated genes were revealed through gene amplification in the HCC samples, we filtered out those DEGs. In addition, significantly downregulated genes resulting from gene deletion in the HCC samples were also removed. As a result, 5,848 upregulated and 1,586 downregulated DEGs in the HCC samples were identified (Additional file 1: Table S7). Furthermore, we examined the transcription level of these DEGs in the two HCC cell lines and found that most of these genes had a similar expression level as in the HCC samples but differed substantially from the non-HCC samples (Additional file 2: Figure S5).

Based on these data, we then examined key genes in the three cancer-related pathways that were enriched in the DMR genes (focal adhesion, Wnt, and MAPK). Notably, the FAK (PTK2), $\beta$-catenin (CTNNB1), and p38 MAPK (MAPK12) genes were all significantly upregulated in HCC. In particular, genes downstream of p38 MAPK including MAPKAPK2, NFkB2, p53, CHOP (DDIT3), MSK (RPS6KA4), CREB (ATF4), and $c-M y c$, were all upregulated, indicating activation of these signaling pathways in the HCC specimens (Figure 3).

\section{TSG candidate screening}

To screen TSG candidates that might be aberrantly regulated by DNA methylation, we cross-matched the selected DEGs with genes that contained DMRs or DhMRs within $5 \mathrm{~kb}$ upstream of their TSS. As $5 \mathrm{hmC}$ is generated by TET proteins via oxidizing $5 \mathrm{mC}[15,16]$, we further required that hypermethylated genes should be hypohydroxymethylated and vice versa, hypomethylated genes should be hyperhydroxymethylated. As a result, expression levels of 124 DMR genes were negatively correlated with their methylation levels, while 22 DhMR genes were positively correlated between their expression and hydroxymethylation levels, in the three matched pairs. Majority (85) of the 124 DMR genes overlapped with the gene set identified in the five 


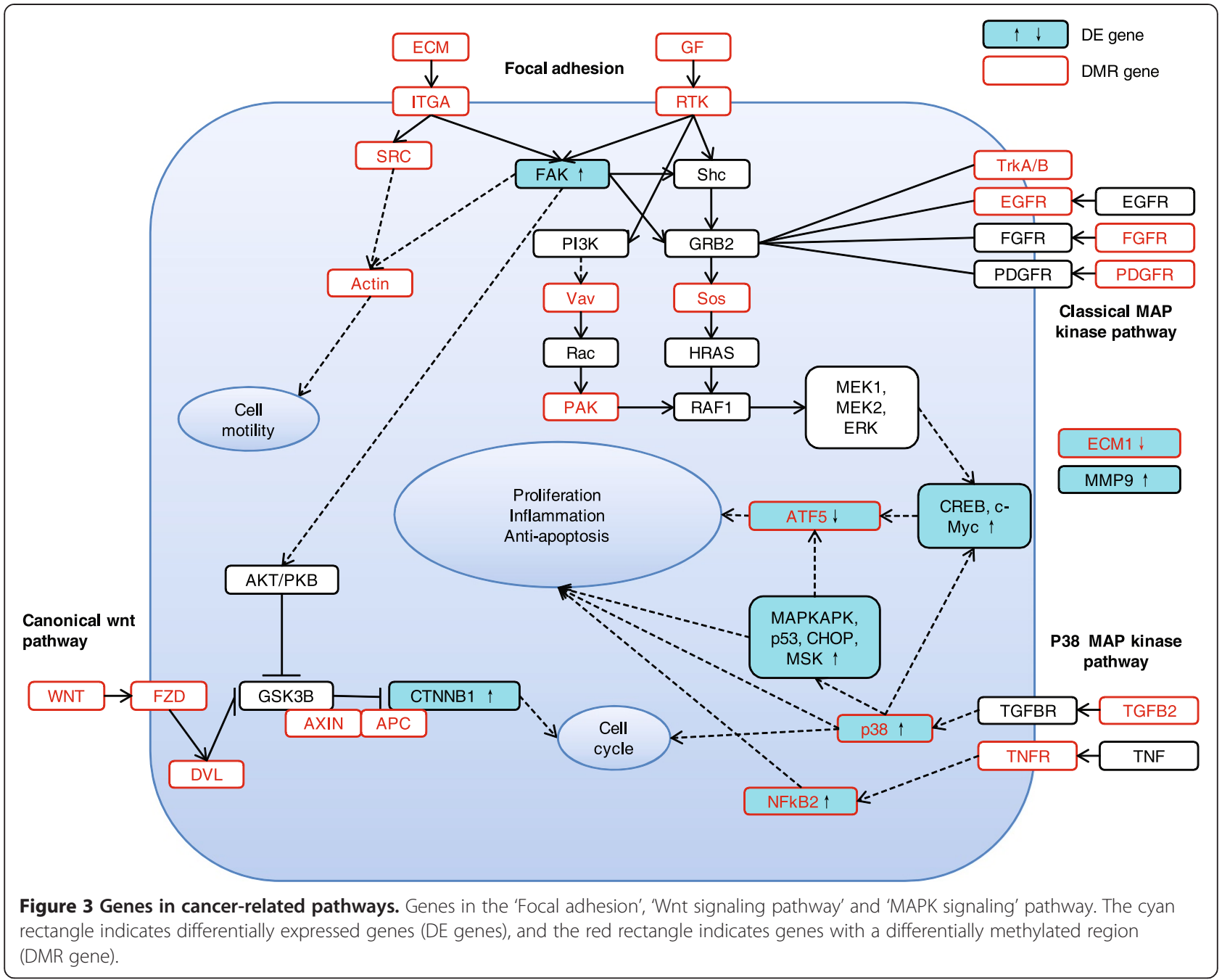

matched pairs. However, only 2 out of the 22 DhMR genes were replicated between the two comparison groups (Additional file 2: Figure S6). These results might indicate that DMRs are more stable than DhMRs in HCC populations. Based on these data and on reports in the literature, which indicated that the biological function of these genes is relevant to liver development or disease, we selected 18 candidate genes for further validation.

Semi-quantitative RT-PCR was then used to detect the mRNA levels of the 18 genes in 20 additional pairs of HCC and non-HCC specimens (Primer sequences listed in Additional file 1: Table S8). Among the 18 genes, six genes including FAM150A, TCF21, EOMES, ATF5, DPT, and $E C M 1$ were frequently downregulated in $85 \%, 85 \%$, $35 \%, 60 \%, 50 \%$, and $60 \%$ of the 20 HCC samples, respectively, compared with non-HCC samples (Additional file 2: Figure S7). As all six of these genes only contained DMRs in their promoters, but not DhMRs, bisulfite sequencing can then efficiently validate DNA methylation changes for these genes. Therefore, we first performed bisulfite sequencing PCR (BSP) on three selected DMR genes (FAM150A, TCF21, and EOMES) in the three pairs of HCC samples for technical validation. The results confirmed significant differential methylation between $\mathrm{HCC}$ and nonHCC samples for each gene, indicating the accuracy of DMR detection by HMST-seq (Additional file 2: Figure S8, Additional file 1: Table S9). Based on these results, we further validated five selected DMR genes (ATF5, ECM1, EOMES, FAM150A, and TCF21) in 20 additional pairs of HCC and non-HCC samples for biological replication. A high-throughput illumina-sequencing-based BSP method that we developed recently was applied [31] (See Methods). Our results confirmed consistently higher methylation levels of all these genes in HCC samples in comparison with nonHCC samples (Figure 4A, Additional file 1: Table S9). In particular, ECM1 and TCF21 displayed significant differential methylation between $\mathrm{HCC}$ and non-HCC groups $(P<0.05)$. Besides of key hypermethylated gene promoters, we also used this method to test four repetitive elements. 


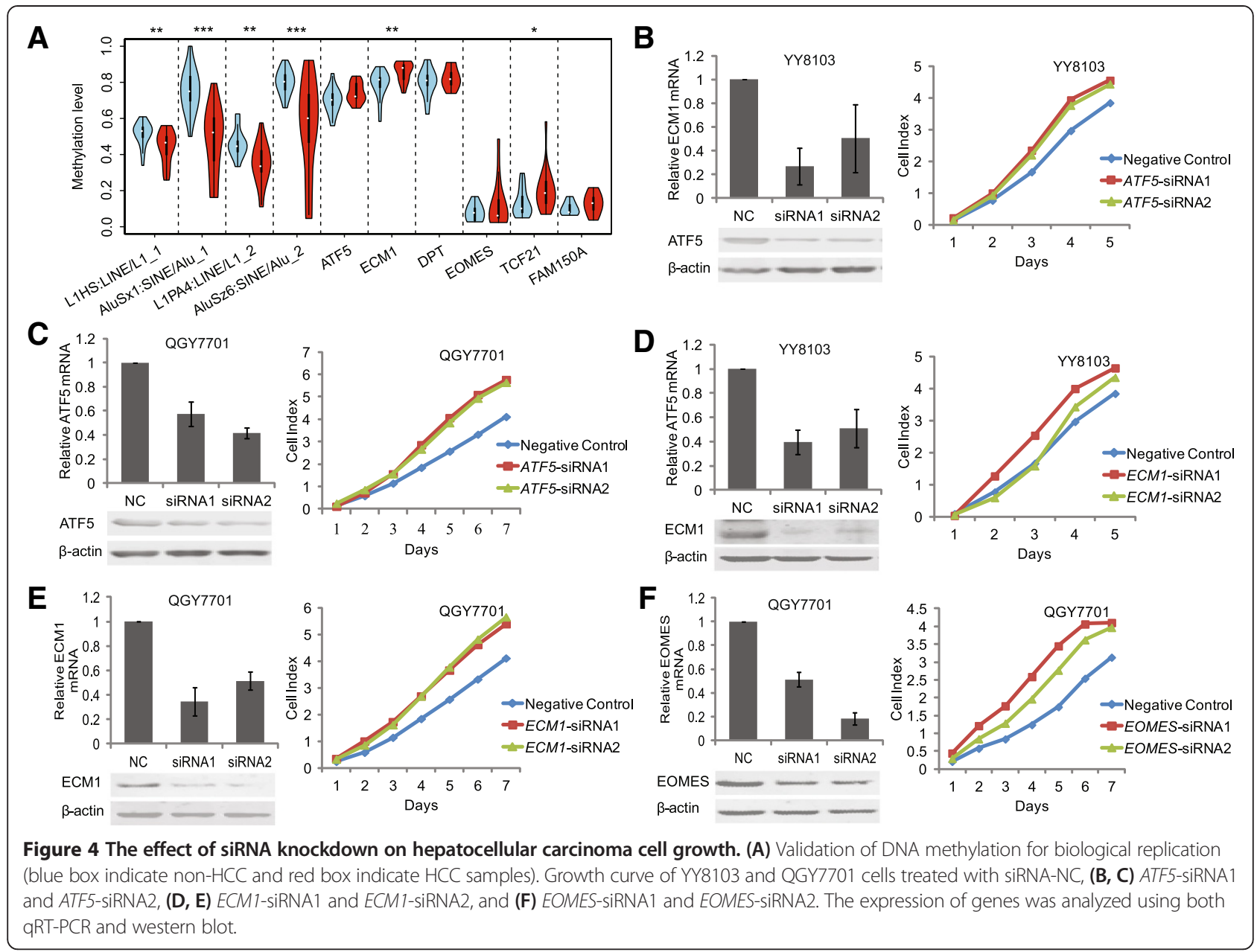

Consistent with whole genome hypomethylation and hypohydroxymethylation tested by UPLC-MS/MS, the methylation levels of these repetitive elements tested by bisulfite sequencing were significantly lower in HCC samples (Figure 4A).

\section{Suppression of endogenous ATF5, ECM1, and EOMES promotes HCC cell line proliferation}

To evaluate the potential contribution of the six target genes to HCC tumorigenesis, we next aimed to perform gene knockdown experiments in HCC cell lines. By evaluating the expression level of six target genes in available HCC cell lines using RT-PCR, we selected YY8103 and QGY7701 HCC cell lines, as in which ATF5, EOMES, and ECM1 were all expressed. However, FAM150A, TCF21, and $D P T$ were hardly expressed in almost all cell lines (Additional file 2: Figure S7). Based on these results, we designed two specific siRNAs against each gene of ATF5, $E O M E S$, and ECM1 (Additional file 1: Table S10) for knockdown experiments, using siRNA-NC as a negative control. Our data showed that the expression of these three endogenous target genes was efficiently knocked down in YY-8103 and QGY7701 cells by these siRNAs, as demonstrated through both real-time PCR assays and western blots (Figure 4B, C, D, E, and F). As a result, the transient transfection of siRNA1 and siRNA2 for these three genes enhanced the growth of YY8103 and QGY7701 HCC cells compared with cells transfected with siRNA-NC $(P<0.05$; Figure 4$)$. Furthermore, analysis of colony formation in soft agar confirmed that among the four target genes, silencing ATF5, ECM1, and EOMES significantly promoted the anchorage-independent growth of YY-8103 and QGY7701 cells compared with the siRNA$\mathrm{NC}$ control cells $(P<0.05$; Figure 5$)$.

Taken together, these data support the hypothesis that knockdown of ATF5, ECM1, and EOMES consistently promoted the growth of existing HCC cells, suggesting that silencing these three genes may contribute to HCC oncogenesis and progression.

\section{Discussion}

Previous studies have shown that hypermethylation of multiple TSGs contributes to HCC pathogenesis and represents a crucial event in HCC progression $[6,9,11,32]$. However, 


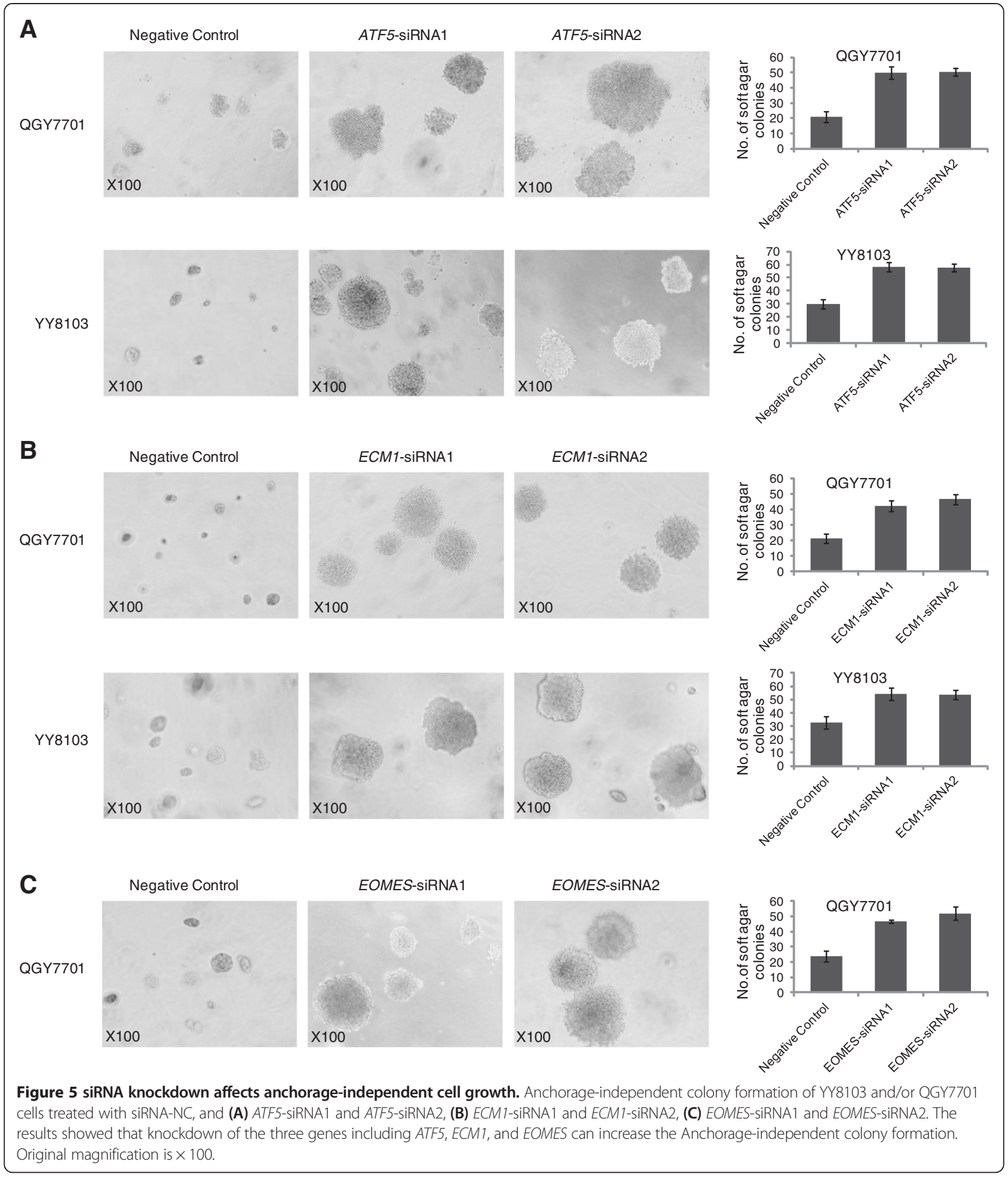

the recently discovered hydroxymethylation has brought an additional dimension of complexity to the methylation alterations observed in cancer. Most previous studies have used bisulfite treatment or methylation-sensitive enzyme digestion-based methods to investigate novel DNA methylation biomarkers, which cannot distinguish 5 -hmC from
5 - $\mathrm{mC}[12,13]$. In addition to the heterogeneity of cancer samples or technological differences, inaccurate quantification of DNA methylation levels might also lead to inconsistent or even contradictory results between different studies [33]. Thus, distinguishing between $5 \mathrm{hmC}$ and $5 \mathrm{mC}$ assists in the identification of DNA methylation biomarkers 
for clinical and commercial use with better sensitivity and specificity.

Here, we applied a multi-omics strategy to vertically integrate epigenomic, genomic, and transcriptomic profiling to screen for novel epigenomic biomarkers and TSG candidates of HCC. We applied both the commonly used UPLC-MS/MS and the newly developed HMST-Seq technologies to simultaneously examine DNA methylation and hydroxymethylation levels. Globally, $5 \mathrm{mC}$ and $5 \mathrm{hmC}$ levels were reduced in HCC, as confirmed by UPLC-MS/ MS. As HMST-Seq can only test CCGG MspI sites, an increasing amount of $5 \mathrm{mC}$ was observed for CCGG sites, in contrast to whole genome $5 \mathrm{mC}$ tendency. This result not only reflects the limitation of HMST-Seq in detection of global $5 \mathrm{mC}$ pattern, but also indicates an uneven distribution of $5 \mathrm{mC}$ in the genome. On the other hand, as $5 \mathrm{hmC}$ were evenly distributed across the genome, consistently low levels of $5 \mathrm{hmC}$ across the genome-wide CCGG MspI sites were also observed. Furthermore, DMRs and DhMRs rarely co-occurred at the same genomic region. These results might support the hypothesis that $5 \mathrm{hmC}$ is an intermediate of the demethylation process $[15,16]$, and loss of hydroxymethylation might be also an epigenetic feature of HCCs [21].

We found that a significant number of DMR genes were enriched in several highly interrelated cancer signaling pathways, including the 'Focal adhesion,' 'Wnt', and 'MAPK' pathways (Figure 2C). Furthermore, the activation of key signaling pathway genes, including $F A K$ in 'Focal adhesion', $\beta$-catenin in 'Wnt', and $p 38$ and its downstream genes in 'MAPK', indicated the activation of these signaling pathways in HCC, which has been previously reported in HCC. Although we currently have no direct evidence that the methylation and hydroxymethylation changes in DMR and DhMR genes may directly contribute to the activation of these pathways, these two types of DNA modifications represent essential participants in heptocarcinogenesis. In addition to gene transcription repression, DNA modifications may also be important for alternative splicing [34], gene mutation [35], and chromatin remodeling [36] in tumorigenesis. Further studies are needed to examine these genes.

In addition to the genes found enriched by KEGG pathway analysis, we confirmed that the suppression of three novel genes by promoter hypermethylation can significantly enhance cell growth and progression in HCC cell lines, indicating their potential as TSG candidates. These three genes have never been reported in previous studies on aberrant DNA methylation of HCCs $[6,9,11,32]$. Of these three genes, ECM1 has been reported to inhibit the activity of matrix metalloproteinase 9 (MMP9) through high-affinity protein/protein interactions [37]. Due to the significant downregulation of ECM1 transcription, our data also support the significant upregulation of $M M P 9$ transcription in
HCC, which agrees with previous observations of highly upregulated MMP expression in many solid tumors [38]. MMPs are key modulators of the tumor microenvironment, and it has been shown that MMPs not only regulate ECM turnover but also cell signaling pathways controlling cell growth, inflammation and angiogenesis in a non-proteolytic manner through various non-catalytic domains [38].

ATF5 is a highly abundant liver-enriched transcription factor that belongs to the ATF/cAMP response elementbinding family [39]. A study has indicated that transcriptional expression of $A T F 5$ is directly induced by $C H O P$ and ATF4, which together with ATF5 form the integrated stress response (ISR) pathway that is critical for alleviating damage accrued during acute stress [40]. Thus, the proapoptotic functions of ATF5 and CHOP have been revealed [40]. In HCC, although $C H O P$ was activated, ATF5 was hypermethylated in its promoter and exhibited downregulated transcriptional expression. These results are in agreement with previous studies that confirmed downregulation of ATF5 transcription [41] and protein expression levels [42] in HCC. Therefore, ATF5 might be blocked in HCC, resulting in antiapoptotic effects and cancer cell survival.

The T-box transcription factor eomesodermin (EOMES) has been found to have a regulatory role for CD8 T cell activity in humans [43] and plays a critical role in tumor immune surveillance and eradication [44]. The absence of $E O M E S$ in tumor-infiltrating lymphocytes correlates with enhanced lymph node metastasis in colorectal cancer [43].

\section{Conclusions}

In summary, ECM1, ATF5, and EOMES may all serve as novel TSGs that are aberrantly hypermethylated at their promoters leading to their downregulated transcription, which results in HCC tumorigenesis and progression. In addition, we have identified three other genes (FAM150A, $T C F 21$, and $D P T$ ) with promoter hypermethylation and significant transcriptional downregulation in HCCs. These six genes may comprise a gene panel that could be used for the clinical diagnosis and prognosis of HCCs with respect to promoter methylation and gene transcription levels.

\section{Materials and methods \\ Sample materials}

All HCC tissue specimens were obtained from patients who underwent surgical resection for their tumors and provided informed consent prior to liver surgery. The primary tumor specimens were immediately frozen at $-80^{\circ} \mathrm{C}$ until DNA/RNA extraction. Specimens (approximately $1 \mathrm{~cm}^{3}$ ) of the tumor and adjacent liver tissue were collected from each patient, and the HCC diagnosis was confirmed through pathological examination. The HCC specimens used in this study were grouped according to 
differentiation grades II to III following the EdmondsonSteiner grading system. The clinical characteristics of the patients and tumors are summarized in Additional file 1: Table S1. This project and protocols involving human and animal tissues were approved by the ethics committee of the Research Ethics Committee of Shenzhen Third People's Hospital (No. 2012-010), and conducted according to the Declaration of Helsinki. The use, for research purposes, of tumor tissues from clinically surgical removal was deemed exempt from a requirement for informed consent beyond the consent normally obtained for this clinical procedure.

Normal liver (including LO2 and WRL68) and HCC cell lines (including Hep3B, SK-hep1, Focus, Huh7, SMMC7721, MHCC97L, MHCC97H, MHCC-LM3, MHCC-LM6, PLC, HepG2, YY8103, QGY7701, QGY7703, BEL7402, BEL7404, and BEL7405) were also used in this study. Of these cell lines, Hep3B, SK-hep1, Focus, Huh7, SMMC7721, PLC, HepG2, YY8103, QGY7701, QGY7703, BEL7402, BEL7404, and BEL7405 were obtained from a commercial source (Institute of Chemistry and Cell Biology at Shanghai). In addition, the MHCC97L, MHCC97H, MHCC-LM3, and MHCC-LM6 cell lines were derived from published references $[45,46]$ and were kindly provided by professor Yinkun Liu from the Liver Cancer Institute affiliated with Zhongshan Hospital in Shanghai.

\section{DNA and RNA extraction and cDNA synthesis}

Genomic DNA was extracted from all samples using the DNeasy Tissue kit (Qiagen, Valencia, CA, USA), according to protocols recommended by the manufacturer. Total RNA was extracted from the HCC cell lines and frozen tissue samples, which were pulverized in liquid nitrogen using TRIzol reagent (Invitrogen, Carlsbad, CA, USA). To reduce the risk of genomic DNA contamination, 1 to $2 \mu \mathrm{g}$ RNA was incubated with $2 \mathrm{U}$ DNase I (Invitrogen, Carlsbad, CA, USA), $1 \mu \mathrm{L}$ DNase buffer and, $0.4 \mu \mathrm{L}$ RNase Out for $15 \mathrm{~min}$ at room temperature. The RNA concentration was determined by spectrophotometry, and the total RNA integrity was examined by visualization of the $28 \mathrm{~S}$ and $18 \mathrm{~S}$ ribosomal RNAs in a $1.2 \%$ agarose gel. First-strand cDNA was synthesized using the PrimeScript RT Reagent Kit (TaKaRa, Otsu, Japan) according to the manufacturer's instructions.

\section{UPLC-MS/MS analysis}

Genomic DNA $(0.2 \mu \mathrm{g})$ extracted from all patients and cell lines was digested with $1 \mathrm{U}$ DNase I, $2 \mathrm{U}$ alkaline phosphatase, calf intestinal, and $0.005 \mathrm{U}$ snake venom phosphodiesterase I at $37^{\circ} \mathrm{C}$ for $24 \mathrm{~h}$. A microcon centrifugal filter device with a 3,000 D cutoff membrane was used to remove protein from the digested DNA samples by centrifuging at $12,000 \mathrm{rpm}$ for $60 \mathrm{~min}$. The mobile phase consisted of $0.1 \%$ formic acid (solvent A) and methanol containing 0.1\% formic acid (solvent B). The flow rate was set to $500 \mu \mathrm{L} /$ $\mathrm{min}$. The enzymatically digested DNA samples $(5 \mu \mathrm{L}$ each) were injected for UPLC-MS/MS analysis with a LC gradient as follows: $0.0-4.0 \mathrm{~min}, 0$ to $50 \%$ of solvent B; $4.0-6.0 \mathrm{~min}$, $50 \%$ solvent B; $6.0-6.1 \mathrm{~min}, 50 \%$ to $5 \%$ of solvent B; and 6.1$15 \mathrm{~min}, 5 \%$ of solvent B. The separated analytes were detected using a 5500 Qtrap linear ion trap quadrupole mass spectrometer equipped with a Turbo $\mathrm{V}$ ion source operated in the ESI mode (AB Sciex) with Analyst software (Version 1.5). The Source and Gas were set as follows: gas 1 , nitrogen (45 psi); gas 2 , nitrogen (40 psi); ion spray voltage, $5,500 \mathrm{~V}$; ion source temperature, $400^{\circ} \mathrm{C}$; and curtain gas, nitrogen (30 psi). The mass spectrometer was operated in the multireaction monitoring mode (MRM).

\section{Data analysis for HSMT-Seq}

Genomic DNA was extracted from all patients and cells using the QIAamp DNA Blood Mini Kit (Qiagen). HSMTSeq library construction was performed following our previously reported protocol [22]. The libraries were sequenced using single-end 50 bp sequencing strategy with an Illumina HiSeq2000 sequencing system. The FASTQ sequence reads were mapped to a virtual human reference library (hg19) with no more than one mismatch after adapter removal and low-quality read filtering. The virtual library was constructed as follows. The human reference genome (hg19) was in silico digested with MspI and NlaIII, and we defined the DNA sequences between the nearest NlaIII sites around each MspI site in both directions as the virtual library reference for mapping. Unambiguous mapped tags were used for further analysis. The data for three libraries were normalized according to a previous method (Global Rank-invariant Set Normalization, GRSN) [47] based on the normalized tag counts, and the modification abundance of a specific CCGG site was determined as the ratio between the tag counts of two libraries. For instance, the hydroxymethylation level can be defined as the ratio between ' $\mathrm{C}+\mathrm{mC}+\mathrm{hmC}$ ' and ' $\mathrm{C}+$ $\mathrm{mC}$ ' tags. Furthermore, CCGG sites with significantly different tag counts (sequencing depth $>10 \mathrm{X}$, FDR $<0.001$ ) based on Poisson distribution with a ratio of tags between two libraries larger than 1 were determined to be significantly modified sites.

For a given genomic interval, the modification frequency of CCGG sites was defined as the ratio of significantly modified sites within all CCGG sites. Differentially methylated or hydroxymethylated regions (DMR or DhMR) between HCC and non-HCC samples were defined in the following steps: (1) The first five CCGG sites containing at least four CCGG sites with the same changing trend and a Wilcoxon ranksum test $P$ value $<0.05$ as a seed site for candidate DMRs was used; (2) a 3' downstream adjacent CCGG with the same changing trend was then incorporated with this candidate DMR. Up to a 2,000-bp inter-distance was allowed between the two adjacent CCGGs, and a Wilcoxon rank-sum 
test was performed in the incorporated region; (3) Repeat these steps until the Wilcoxon rank-sum test $P$ value is $\geq 0.05$; and (4) the incorporated region was defined as a DMR or DhMR.

\section{Enrichment analysis}

Functional enrichment analysis for genes with DMRs or DhMRs was performed using WebGestalt [48,49], which is freely accessible at [50].

\section{Data analysis for differentially expressed genes (DEGs)}

The gene expression levels based on RNA-seq and Affymetrix microarray data were measured by the reads per kilobase of transcript per million reads (RPKM) and tags per million reads (TPM) algorithm, respectively. The differentially expressed genes (DEGs) were identified in the five pair-wise samples with Affymetrix microarray data by Wilcoxon rank-sum test and in each of the two pairwise samples with RNA-seq data according to a previously published method [51]. Genes that were significantly expressed (FDR <0.05) in at least one group and regulated similarly in all the groups were further identified as differentially expressed genes (DEGs).

\section{Illumina-sequencing-based BSP}

Illumina-sequencing-based BSP was performed based on a previously described strategy with minor adjustment [31]. PCR primers were designed using online software [52] and then concatenated 22 bp common sequences at their 5' end, respectively, which would mediate high throughput sequencing linker extension during the following PCR procedure. All PCR products were about $500 \mathrm{bp}$. For the high throughput bisulfite-amplicon library construction using large scale of clinical samples, WaferGen Biosystem (WaferGenBiosystems) was employed. Briefly, $500 \mathrm{ng}$ of genomic DNA extracted from whole blood were converted using EZ DNA Methylation-Gold Kit ${ }^{\mathrm{m}}$ (ZYMO). For each sample, bisulfite converted genomic DNA, KAPA2G Robust HotStart ReadyMix (KAPA Biosystems), PCR primers, PCR primers mediated high throughput sequencing linkers with a barcode sequence were dispensed into one individual nanowell of a Smart MyDesign Chip, which contains 5,184 nanowells, by Smart Chip Multisample Nanodispenser. Thirty-six amplicons for each sample and 150 samples could be constructed as one library with barcode sequence classifying samples using one Smart MyDesign Chip. PCR amplification of Smart MyDesign Chip was conducted using the Techne Prime Thermal Cycler. PCR product was purified using the QIAquick Gel Extraction Kit (Qiagen). After analyzed by an Agilent 2100 Bioanalyzer (Agilent Technologies) and quantified by real-time PCR, the library was sequenced with pair end $250 \mathrm{bp}$ using Illumina Miseq sequencer.

\section{Semi-quantitative RT-PCR}

Reverse transcription (RT) was performed with $2 \mu \mathrm{g}$ of total RNA treated with RNase-free DNase I. Semiquantitative RT-PCR was then performed using primers for target genes (Additional file 1: Table S8). The length of the amplified fragments was $350 \mathrm{bp}$, and $\beta$-actin served as an internal reference. The primers for $\beta$-actin were as follows: 5'-TCACCCACACTGTGCCCATC TACGA-3' (forward) and 5'-CAGCGGAACCGCT CATTGCCAATGG-3' (reverse). The length of the $\beta$ actin amplicon was $295 \mathrm{bp}$. The PCR products were separated in $2 \%$ agarose gels containing ethidium bromide. The PCR reactions were performed in a volume of $20 \mu \mathrm{L}$ using the TaKaRa PCR Kit. The reactions were performed at $94^{\circ} \mathrm{C}$ for $5 \mathrm{~min}$ followed by 30 to 35 cycles (for 18 target genes) or 25 cycles (for $\beta$-actin) at $94^{\circ} \mathrm{C}$ for $30 \mathrm{~s}, 55^{\circ} \mathrm{C}$ for $30 \mathrm{~s}$, and $72^{\circ} \mathrm{C}$ for $30 \mathrm{~s}$ with a final extension at $70^{\circ} \mathrm{C}$ for $5 \mathrm{~min}$. The $\mathrm{PCR}$ products were brought to $4^{\circ} \mathrm{C}$ at the end of the reaction, and they were then separated in $2 \%$ agarose gels containing ethidium bromide.

\section{Western blot}

Cells were collected using a $2 \times$ loading lysis buffer $(2 \times$ concentrations: $50 \mathrm{mmol} / \mathrm{L}$ Tris- $\mathrm{HCl}, \mathrm{pH}$ 6.8, 2\% sodium dodecyl sulfate, 10\% 2-mercaptoethanol, 10\% glycerol, and $0.002 \%$ bromophenol blue). Total protein extracts from cultured cells were subjected to protein gel electrophoresis using 12\% SDS-PAGE, transferred to a HybridPVDA membrane (Amersham Life Sciences) via a semidry electrophoretic transfer method, and treated with $20 \%$ methanol in a Tris-glycine buffer $(25 \mathrm{mM}$ Tris- $\mathrm{HCl}$, $\mathrm{pH}$ 8.0, 0.2 M glycine, 0.1\% SDS). After blocking with PBS containing 5\% BSA, the membrane was incubated for immunoblotting analysis with a rabbit anti-myc polyclonal antibody (1:100; made in our laboratory) or antimyc antibody $(1: 1,000)$ at room temperature for $2 \mathrm{~h}$, followed by incubation with an IRDye 800DXconjugated, affinity-purified goat anti-rabbit secondary antibody (1:1,000; Rockland). Signals were detected using the Odyssey Infrared Imaging System (LI-COR Biosciences). $\beta$-actin was used as a loading control.

\section{Identification of CNVs}

Array-based comparative genomic hybridization (aCGH) was performed using Agilent 244-k arrays as previously described [23]. CNVs that were present in the matching normal sample were filtered, and the remaining CNVs were regarded as somatic variants.

\section{RNA interference}

Three siRNAs directed against five target genes were designed on the Whitehead Institute Web Server (http:// jura.wi.mit.edu/bioc/siRNAext/) and chemically synthesized 
(Shanghai GenePharma Co.) to target the different coding regions of each gene. The siRNA sequences are shown in Additional file 1: Table S6. In addition, siRNA-NC (5'GAGUUAAAGUCAAAGUGACTT- $3^{\prime}$ and $5^{\prime}$-GUCACU UUGACUUUAACUCTT-3') was also synthesized. The siRNAs were transfected into the HCC cell lines, and cell growth was monitored. For siRNA transfection, $3 \times 10^{3}$ HCC cells per well were seeded into 96-well plates. When the cells reached $30 \%$ to $50 \%$ confluence, they were transfected with synthetic siRNAs at a final concentration of 50 nM using the Lipofectamine 2000 Transfection Reagent (Invitrogen) according to the manufacturer's instructions.

\section{Cell proliferation analysis}

The cells were cultured for 7 days, and cell viability was measured using the ACEA RTCA kit (ACEA Biosciences, San Diego, CA, USA). A microelectronic cell sensor system was used to confirm the number of living cells. NSCLC cells $\left(1 \times 10^{4}\right)$ were seeded into each sensor-containing well (19.6- $\mathrm{mm}^{2}$ surface with $150 \mathrm{~mL}$ of medium) of the microtiter plates. The electronic sensors provided a continuous (every $6 \mathrm{~h}$ ), quantitative measurement of the cell index (reflecting the surface area covered by the cells) in each well. Cell growth was measured every $6 \mathrm{~h}$ for 96 to $144 \mathrm{~h}$, and cell indexes were recorded for each well at all time points to assess cell viability. All experiments were independently repeated at least three times.

\section{Soft agar colony formation}

For soft agar colony formation assays in 24-well plates, $2 \times 10^{4}$ cells were plated and grown on plates containing $1 \%$ base agar and $0.5 \%$ top agar. The plated cells were incubated at $37^{\circ} \mathrm{C}$ for 21 days. The plates were stained with $0.005 \%$ crystal violet for $1 \mathrm{~h}$, and colonies were counted under a dissecting microscope. All experiments were independently repeated at least three times.

\section{Data availability}

The sequencing and processed data have been deposited in the NCBI SRA and GEO database under the accession number SRA092219 and GSE54141. All the UPLC-MS/MS raw data were deposited in figshare database (http://dx.doi. org $/ 10.6084 / \mathrm{m} 9$.figshare.1117723).

\section{Additional files}

Additional file 1: All supplemental tables (Tables S1 to S10) and corresponding captions.

Additional file 2: All supplemental figures (Figures S1 to S8) and corresponding legends.

\section{Abbreviations}

5fC: 5-formylcytosine; 5hmC: 5-hydroxymethylcytosine; 5mC: 5-methylcytosine; CNV: Copy number variation; DEGs: Differentially expressed genes;

DhMRs: Differentially hydroxymethylated regions; DMRs: Differentially methylated regions; HCC: Hepatocellular carcinoma; HMST-seq: Hydroxymethylation and methylation Sensitive Tag sequencing; non-HCC: Non-cancerous liver tissues; TSG: Tumor suppressor gene; TSS: Transcriptional start sites; UPLC-MS/MS: Ultra-performance liquid chromatography/tandem mass spectrometry.

\section{Competing interests}

The authors declare that they have no competing interests.

\section{Authors' contributions}

$\mathrm{FG}, J \mathrm{H}$, and $\mathrm{XQZ}$ conceived the project; $Y \mathrm{YX}$ conducted the bioinformatic analysis; JWW and HJL performed the HMST-Seq experiments; YO, WL, BPZ, and $X L$ prepared samples and performed the functional experiments; and ZLL, BJZ, and BW performed the UPLC-MS/MS experiment. FG, YDX, and JH interpreted the data and wrote the manuscript. All authors read and approved the final manuscript.

\section{Acknowledgments}

We gratefully acknowledge support from the National High Technology Research and Development Program of China (863 Program, 2012AA02A201 and 2012AA02A205), the Chinese National Key Program on Basic Research (973 Program, 2010 CB529206 and 2014CB965002), the National Natural Science Foundation of China (81272306), Program of Shanghai Subject Chief Scientist (12XD1421400), Guangdong Innovative Research Team Program (2009010016), and Program of Shenzhen Commission for Science and Technology (JCYJ20130329171031740).

\section{Author details}

${ }^{1}$ BGl-Shenzhen, Shenzhen 518083, China. ${ }^{2}$ National Engineering Center for Biochip at Shanghai, Shanghai 201203, China. ${ }^{3}$ Shanghai-MOST Key Laboratory for Disease and Health Genomics, Chinese National Human Genome Center at Shanghai, Shanghai 201203, China. ${ }^{4}$ Shenzhen Key Lab. of Infection and Immunity, Shenzhen Third People's Hospital, Guangdong Medical College, Shenzhen 518112, China. ${ }^{5}$ Guangdong Key Lab. Of Diagnosis \& Treatment for Emerging Infectious Disease, Shenzhen Third People's Hospital, Guangdong Medical college, Shenzhen 518112, China.

Received: 14 April 2014 Accepted: 6 November 2014

Published online: 03 December 2014

\section{References}

1. Llovet JM, Burroughs A, Bruix J: Hepatocellular carcinoma. Lancet 2003, 362:1907-1917.

2. Ferlay J, Shin HR, Bray F, Forman D, Mathers C, Parkin DM: Estimates of worldwide burden of cancer in 2008: GLOBOCAN 2008. Int J Cancer 2010, 127:2893-2917.

3. Sherman M: Recurrence of hepatocellular carcinoma. N Engl J Med 2008, 359:2045-2047.

4. Nishida N, Goel A: Genetic and epigenetic signatures in human hepatocellular carcinoma: a systematic review. Curr Genomics 2011, 12:130-137.

5. Liu M, Jiang L, Guan XY: The genetic and epigenetic alterations in human hepatocellular carcinoma: a recent update. Cell Protein 2014, 5:673-691.

6. Anwar SL, Lehmann U: DNA methylation, microRNAs, and their crosstalk as potential biomarkers in hepatocellular carcinoma. World I Gastroenterol 2014, 20:7894-7913.

7. Eden A, Gaudet F, Waghmare A, Jaenisch R: Chromosomal instability and tumors promoted by DNA hypomethylation. Science 2003, 300:455.

8. Chan KC, Jiang P, Chan CW, Sun K, Wong J, Hui EP, Chan SL, Chan WC, Hui DS, Ng SS, Chan HLY, Wong CSC, Ma BBY, Chan ATC, Lai PBS, Sun H, Chiu RWK, Lo YMD: Noninvasive detection of cancer-associated genome-wide hypomethylation and copy number aberrations by plasma DNA bisulfite sequencing. Proc Natl Acad Sci U S A 2013, 110:18761-18768.

9. Nishida N, Kudo M, Nagasaka T, Ikai I, Goel A: Characteristic patterns of altered DNA methylation predict emergence of human hepatocellular carcinoma. Hepatology 2012, 56:994-1003.

10. Umer M, Qureshi SA, Hashmi ZY, Raza A, Ahmad J, Rahman M, lqbal M: Promoter hypermethylation of Wnt pathway inhibitors in hepatitis $C$ virus - induced multistep hepatocarcinogenesis. Virol J 2014, 11:117.

11. Shen J, Wang S, Zhang YJ, Wu HC, Kibriya MG, Jasmine F, Ahsan H, Wu DP, Siegel $A B$, Remotti $H$, Santella RM: Exploring genome-wide DNA methylation profiles altered in hepatocellular carcinoma using Infinium HumanMethylation 450 BeadChips. Epigenetics 2013, 8:34-43.

12. Huang Y, Pastor WA, Shen Y, Tahiliani M, Liu DR, Rao A: The behaviour of 5-hydroxymethylcytosine in bisulfite sequencing. PLOS One 2010, 5:e8888. 
13. Jin SG, Kadam S, Pfeifer GP: Examination of the specificity of DNA methylation profiling techniques towards 5-methylcytosine and 5-hydroxymethylcytosine. Nucleic Acids Res 2010, 38:e125.

14. Gao F, Xia Y: Hydroxymethylation- and methylation-sensitive tag sequencing: how will this technology change clinical applications of DNA methylation profiling? Epigenomics 2013, 5:355-357.

15. Tahiliani M, Koh KP, Shen Y, Pastor WA, Bandukwala H, Brudno Y, Agarwal S, lyer LM, Liu DR, Aravind L, Rao A: Conversion of 5-methylcytosine to 5-hydroxymethylcytosine in mammalian DNA by MLL partner TET1. science 2009, 324:930-935

16. Ito S, Shen L, Dai Q, Wu SC, Collins LB, Swenberg JA, He C, Zhang Y: Tet proteins can convert 5-methylcytosine to 5-formylcytosine and 5-carboxylcytosine. Science 2011, 333:1300-1303.

17. Kriaucionis S, Heintz N: The nuclear DNA base 5-hydroxymethylcytosine is present in Purkinje neurons and the brain. Science 2009, 324:929-930.

18. Haffner MC, Chaux A, Meeker AK, Esopi DM, Gerber J, Pellakuru LG, Toubaji A, Argani P, lacobuzio-Donahue C, Nelson WG, Netto GJ, De Marzo AM, Yegnasubramanian S: Global 5-hydroxymethylcytosine content is significantly reduced in tissue stem/progenitor cell compartments and in human cancers. Oncotarget 2011, 2:627-637.

19. Yang H, Liu Y, Bai F, Zhang JY, Ma SH, Liu J, Xu ZD, Zhu HG, Ling ZQ, Ye D, Guan KL, Xiong Y: Tumor development is associated with decrease of TET gene expression and 5-methylcytosine hydroxylation. Oncogene 2013, 32:663-669.

20. Lian CG, Xu Y, Ceol C, Wu F, Larson A, Dresser K, Xu W, Tan L, Hu Y, Zhan Q, Lee CW, Hu D, Lian BQ, Kleffel S, Yang Y, Neiswender J, Khorasani AJ, Fang R, Lezcano C, Duncan LM, Scolyer RA, Thompson JF, Kavakand H, Houvras Y, Zon LI, Mihm MC Jr, Kaiser UB, Schatton T, Woda BA, Murphy GF, et al: Loss of 5-hydroxymethylcytosine is an epigenetic hallmark of melanoma. Cell 2012, 150:1135-1146.

21. Liu C, Liu L, Chen X, Shen J, Shan J, Xu Y, Yang Z, Wu L, Xia F, Bie P, Cui Y, Bian XW, Qian C: Decrease of 5-hydroxymethylcytosine is associated with progression of hepatocellular carcinoma through downregulation of TET1. PLoS One 2013, 8:e62828.

22. Gao F, Xia Y, Wang J, Luo H, Gao Z, Han X, Zhang J, Huang X, Yao Y, Lu H, Yi N, Zhou B, Lin Z, Wen B, Zhang $X$, Yang $H$, Wang J: Integrated detection of both 5-mC and 5-hmC by high-throughput tag sequencing technology highlights methylation reprogramming of bivalent genes during cellular differentiation. Epigenetics 2013, 8:421-430.

23. Bostick M, Kim JK, Esteve PO, Clark A, Pradhan S, Jacobsen SE: UHRF1 plays a role in maintaining DNA methylation in mammalian cells. Science 2007, 317:1760-1764.

24. Bronner C, Krifa M, Mousli M: Increasing role of UHRF1 in the reading and inheritance of the epigenetic code as well as in tumorogenesis. Biochem Pharmacol 2013, 86:1643-1649.

25. Huang J, Deng Q, Wang Q, Li KY, Dai JH, Li N, Zhu ZD, Zhou B, Liu XY, Liu RF, Fei QL, Chen H, Cai B, Zhou B, Xiao HS, Qin LX, Han ZG: Exome sequencing of hepatitis $B$ virus-associated hepatocellular carcinoma. Nat Genet 2012, 44:1117-1121.

26. Romer LH, Birukov KG, Garcia JG: Focal adhesions: paradigm for a signaling nexus. Circ Res 2006, 98:606-616.

27. Zhao J, Guan JL: Signal transduction by focal adhesion kinase in cancer. Cancer Metastasis Rev 2009, 28:35-49.

28. Fatima S, Lee NP, Luk JM: Dickkopfs and Wnt/beta-catenin signalling in liver cancer. World J Clin Oncol 2011, 2:311-325.

29. Christofk HR, Vander Heiden MG, Harris MH, Ramanathan A, Gerszten RE, Wei R, Fleming MD, Schreiber SL, Cantley LC: The M2 splice isoform of pyruvate kinase is important for cancer metabolism and tumour growth. Nature 2008, 452:230-233.

30. Dong C, Yuan T, Wu Y, Wang Y, Fan TW, Miriyala S, Lin Y, Yao J, Shi J, Kang T, Lorkiewicz P, St Clair D, Hung MC, Evers BM, Zhou BP: Loss of FBP1 by Snail-mediated repression provides metabolic advantages in basal-like breast cancer. Cancer Cell 2013, 23:316-331.

31. Gao F, Zhang J, Jiang P, Gong D, Wang JW, Xia Y, Ostergaard MV, Wang J, Sangild PT: Marked methylation changes in intestinal genes during the perinatal period of preterm neonates. BMC Genomics 2014, 15:716.

32. Neumann $O$, Kesselmeier M, Geffers R, Pellegrino R, Radlwimmer B, Hoffmann K, Ehemann V, Schemmer P, Schirmacher P, Lorenzo Bermejo J, Longerich T: Methylome analysis and integrative profiling of human HCCs identify novel protumorigenic factors. Hepatology 2012, 56:1817-1827.
33. Heyn $H$, Esteller M: DNA methylation profiling in the clinic: applications and challenges. Nat Rev Genet 2012, 13:679-692.

34. Maunakea AK, Chepelev I, Cui K, Zhao K: Intragenic DNA methylation modulates alternative splicing by recruiting MeCP2 to promote exon recognition. Cell Res 2013, 23:1256-1269.

35. Hitchins MP, Rapkins RW, Kwok CT, Srivastava S, Wong JJ, Khachigian LM Polly P, Goldblatt J, Ward RL: Dominantly inherited constitutional epigenetic silencing of MLH1 in a cancer-affected family is linked to a single nucleotide variant within the 5'UTR. Cancer Cell 2011, 20:200-213.

36. Forn M, Munoz M, Tauriello DV, Merlos-Suarez A, Rodilla V, Bigas A, Batlle E, Jorda M, Peinado MA: Long range epigenetic silencing is a trans-species mechanism that results in cancer specific deregulation by overriding the chromatin domains of normal cells. Mol Oncol 2013, 7:1129-1141.

37. Fujimoto N, Terlizzi J, Aho S, Brittingham R, Fertala A, Oyama N, McGrath JA, Uitto J: Extracellular matrix protein 1 inhibits the activity of matrix metalloproteinase 9 through high-affinity protein/protein interactions. Exp Dermatol 2006, 15:300-307.

38. Kessenbrock K, Plaks V, Werb Z: Matrix metalloproteinases: regulators of the tumor microenvironment. Cell 2010, 141:52-67.

39. Pascual M, Gomez-Lechon MJ, Castell JV, Jover R: ATF5 is a highly abundant liver-enriched transcription factor that cooperates with constitutive androstane receptor in the transactivation of CYP2B6: implications in hepatic stress responses. Drug Metab Dispos 2008, 36:1063-1072.

40. Teske BF, Fusakio ME, Zhou D, Shan J, McClintick JN, Kilberg MS, Wek RC: CHOP induces activating transcription factor 5 (ATF5) to trigger apoptosis in response to perturbations in protein homeostasis. Mol Biol Cell 2013, 24:2477-2490.

41. Gho JW, Ip WK, Chan KY, Law PT, Lai PB, Wong N: Re-expression of transcription factor ATF5 in hepatocellular carcinoma induces G2-M arrest. Cancer Res 2008, 68:6743-6751.

42. Liu X, Liu D, Qian D, Dai J, An Y, Jiang S, Stanley B, Yang J, Wang B, Liu DX: Nucleophosmin (NPM1/B23) interacts with activating transcription factor 5 (ATF5) protein and promotes proteasome- and caspase-dependent ATF5 degradation in hepatocellular carcinoma cells. J Biol Chem 2012, 287:19599-19609.

43. Atreya I, Schimanski CC, Becker C, Wirtz S, Dornhoff H, Schnurer E, Berger MR, Galle PR, Herr W, Neurath MF: The T-box transcription factor eomesodermin controls CD8 T cell activity and lymph node metastasis in human colorectal cancer. Gut 2007, 56:1572-1578.

44. Zhu Y, Ju S, Chen E, Dai S, Li C, Morel P, Liu L, Zhang X, Lu B: T-bet and eomesodermin are required for $T$ cell-mediated antitumor immune responses. J Immunol 2010, 185:3174-3183.

45. Tian J, Tang ZY, Ye SL, Liu YK, Lin ZY, Chen J, Xue Q: New human hepatocellular carcinoma (HCC) cell line with highly metastatic potential (MHCC97) and its expressions of the factors associated with metastasis. Br J Cancer 1999, 81:814-821.

46. Li Y, Tang Z, Ye S, Liu Y, Chen J, Xue Q, Huang X, Bao W, Yang J, Gao D: [Establishment of human hepatocellular carcinoma cell line with spontaneous pulmonary metastasis through in vivo selection]. Zhonghua Yi Xue Za Zhi 2002, 82:601-605.

47. Pelz CR, Kulesz-Martin M, Bagby G, Sears RC: Global rank-invariant set normalization (GRSN) to reduce systematic distortions in microarray data. BMC Bioinformatics 2008, 9:520.

48. Zhang B, Kirov S, Snoddy J: WebGestalt: an integrated system for exploring gene sets in various biological contexts. Nucleic Acids Res 2005, 33:W741-W748

49. Wang J, Duncan D, Shi Z, Zhang B: WEB-based GEne SeT AnaLysis Toolkit (WebGestalt): update 2013. Nucleic Acids Res 2013, 41:W77-W83.

50. WebGestalt. [http://www.webgestalt.org]

51. Stéphane Audic J-MC: The significance of digital gene expression profiles. Genome Res 1997, 7:986-995.

52. MethPrimer. [www.urogene.org/methprimer/index.html]

\section{doi:10.1186/s13059-014-0533-9}

Cite this article as: Gao et al:: Integrated analyses of DNA methylation and hydroxymethylation reveal tumor suppressive roles of ECM1, ATF5, and EOMES in human hepatocellular carcinoma. Genome Biology 2014 15:533. 